\title{
e-Migrinter
}

9| 2012

Immigrés, illégaux, réfugiés. Questions sur les enquêtes et les catégories

\section{La construction de la catégorie "réfugié » dans un camp en R.D.C. : rôle de l'institution, stratégies des exilés et place du chercheur}

\section{Virginie Tallio}

\section{OpenEdition}

\section{Journals}

Édition électronique

URL : https://journals.openedition.org/e-migrinter/757

DOI : 10.4000/e-migrinter.757

ISSN : 1961-9685

Éditeur

UMR 7301 - Migrinter

Édition imprimée

Date de publication : 20 juillet 2012

Pagination : 18-28

ISSN : 1961-9685

Référence électronique

Virginie Tallio, «La construction de la catégorie « réfugié » dans un camp en R.D.C. : rôle de

l'institution, stratégies des exilés et place du chercheur », e-Migrinter [En ligne], 9 | 2012, mis en ligne le , consulté le 20 mai 2021. URL : http://journals.openedition.org/e-migrinter/757 ; DOI : https://doi.org/ 10.4000/e-migrinter.757 


\section{La construction de la catégorie " réfugié " dans un camp en R.D.C. : rôle de l'institution, stratégies des exilés et place du chercheur}

Virginie Tallio

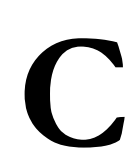

et article est basé sur les résultats d'une enquête de terrain réalisée dans un camp en République Démocratique du Congo (RDC). Il porte sur les conditions de travail du chercheur dans ce contexte. En effet, différents paramètres sont à prendre en compte. Tout d'abord, la présence des institutions gérant le camp modifie la relation entre le chercheur et son objet d'enquête. De plus, la sensibilité du terrain, sensibilité qui se décline sur plusieurs registres, modèle la collecte de données et son interprétation.

\section{Introduction}

Les recherches menées sur les réfugiés vivant en camps se caractérisent à la fois par une présence institutionnelle forte avec laquelle le chercheur doit composer et par une certaine "sensibilité » intrinsèque au terrain. Tout d'abord, l'institution est partie prenante de la recherche, notamment parce qu'elle conditionne l'accès au terrain. Avec l'introduction de ce nouveau protagoniste, la relation entre l'objet d'enquête et le chercheur n'est plus duale, mais triangulaire. De plus, la « sensibilité » de ce terrain peut se définir de plusieurs manières, par la souffrance endurée par la population lors de la fuite, par leurs conditions de vie actuelles, par les enjeux socio-politiques inhérents à ce type de contexte et enfin, par l'adaptation constante des méthodes classiques de recueil des données que cela implique. Le HautCommissariat aux Réfugiés des Nations Unies (H.C.R.) est l'organisme central autour duquel s'articule l'organisation des camps. Il crée la catégorie de population "réfugié ", notamment en la confinant dans un lieu particulier, le camp, puis en organisant les conditions de leur (sur)vie. Il est aussi omniprésent dans l'organisation logistique de l'enquête de terrain.

Comment alors travailler en tant que chercheur dans un tel contexte? Tout d'abord, nous aborderons les conditions de la mise en place de la catégorie «réfugié » et son utilisation tant par les agences humanitaires que par les réfugiés euxmêmes, dans un premier temps en termes spatiaux puis, dans un second temps, en termes administratifs. Ensuite, dans un troisième temps, nous examinerons la place particulière qu'occupe le chercheur dans 
cette configuration triangulaire et les implications méthodologiques que cette configuration implique. Enfin, dans un quatrième temps, il sera question de la triple sensibilité de ce terrain de recherche, ses conséquences sur le recueil de données et notamment ce qui se joue autour de la recherche de la "vérité » et de la «confiance». Pour illustrer nos propos, nous nous baserons sur les résultats d'une enquête de terrain réalisée dans le camp de Nkondo en République Démocratique du Congo (R.D.C.) qui a accueilli des populations angolaises jusqu'en décembre $2004^{1}$.

\section{La spatialisation de la catégorie " réfugié "}

L'Angola sort d'un conflit qui a duré plus de trente ans, puisqu'à une guerre d'indépendance qui s'est déroulée de 1961 à 1975 a succédé une guerre civile qui prit fin en $2002^{2}$. Un million de personnes ont trouvé la mort lors de ce conflit, 500000 personnes ont fui le pays pour chercher asile à l'extérieur et plus de 4 millions ont été forcés de se déplacer à l'intérieur de l'Angola pour tenter de trouver de meilleures conditions de vie (Richardson, 2002). Durant ces années de guerre, les relations avec les pays voisins, c'est-à-dire la R.D.C., la Zambie et la Namibie, ont été marquées par les allers-et-venues des réfugiés angolais. Certains se sont intégrés au sein de la population locale, d'autres dans des camps que le H.C.R. a mis en place le long de la frontière avec la R.D.C. ${ }^{3}$ La province du

${ }^{1}$ Cette recherche s'est déroulée d'août à décembre 2003. Elle a été effectuée dans le cadre d'une thèse de doctorat en anthropologie sociale et ethnologie, soutenue sous la direction de Michel Agier en mai 2007 à l'École des Hautes Études en Sciences Sociales.

${ }^{2}$ Depuis 2002, la paix semble relativement stable. Les élections de septembre 2008, largement gagnées par le Movimento Popular de Libertação de Angola (M.P.L.A.), ont conforté José Eduardo dos Santos dans son fauteuil de président, qu'il occupe depuis 1978, et ont confirmé sa mainmise sur le pays.

${ }^{3}$ D'autres camps ouverts aux réfugiés angolais ont
Bas-Congo, en R.D.C., a accueilli plusieurs de ces camps, notamment ceux de Nkondo et Kilueka ${ }^{4}$. Le camp de Kilueka fut créé le 10 mai 1999 suite à l'arrivée massive de réfugiés angolais fuyant la reprise des combats dans leur pays entre le Movimento Popular de Libertação de Angola (M.P.L.A.) et l'União para a Independência Total de Angola (U.N.I.T.A.). Quant au camp de Nkondo, il fut créé le 3 septembre 1999 quand celui de Kilueka ne fut plus en mesure de recevoir de nouveaux arrivants. En 2002, après la signature d'accords de paix, les réfugiés commencèrent à regagner spontanément leur pays. En décembre 2002, un accord tripartite fut trouvé entre le gouvernement angolais, le gouvernement congolais et le H.C.R., afin de permettre le rapatriement des réfugiés établis dans les camps gérés par ce dernier. Ce rapatriement commença dès août 2003 et suivit un processus législatif complexe.

Le camp de Nkondo a été établi dans la province du Bas-Congo près de la ville de Kimpese qui compte 40000 habitants. Sa situation entre l'Angola et le CongoBrazzaville lui confère une place de carrefour économique. Le camp est néanmoins dans une zone rurale dont la principale ressource est l'agriculture vivrière. La terre qu'il occupe appartient au village de Mpete, situé à quelques centaines de mètres de son entrée. Le site d'implantation a été choisi en fonction de certains critères tels que la proximité d'un point d'eau et la disponibilité en bois de chauffe et de terres arables. Un autre critère de sélection de ce site a été la présence de nombreux Angolais dans le village de Mpete et dans ceux des environs. Selon le H.C.R., la présence de compatriotes devait faciliter l'intégration de cette nouvelle vague de réfugiés et leur acceptation par la population locale, même si certains réfugiés du camp de Nkondo avaient déjà vécu en R.D.C. Nkondo a

aussi été créés en Namibie et en Zambie.

${ }^{4} \mathrm{Je}$ renvoie à l'article de Jacob Sabakinu (2004) pour un historique de la présence des Angolais en R.D.C.. 
compté jusqu'à plus de 5000 réfugiés ${ }^{5}$ majoritairement issus des provinces de Uige et de Zaïre. Instituée par le H.C.R., la gestion en a été déléguée à l'organisation non gouvernementale (O.N.G.) International Rescue Committee (I.R.C.). D'autres agences humanitaires interviennent dans le camp. Elles ont mis en place les infrastructures permettant d'assurer les besoins vitaux de la population: distribution de nourriture, scolarisation des enfants et soins médicaux.

Le camp est partagé en vingt cellules qui regroupent chacune une dizaine d'habitations, des cases en branchages ou des maisons en terre battue, très rarement en briques. Chaque cellule est dirigée par un Comité ${ }^{6}$ qui comprend un cornador, chef de cellule et neuf personnes, ainsi que des «sages", réfugiés dont les qualités diplomatiques et le jugement sûr sont reconnus par la communauté. Ces Comités élisent ensuite le Comité des Réfugiés qui constitue l'organe central. Leurs assemblées sont des lieux de discussion où les réfugiés abordent les problèmes qu'ils rencontrent. Le H.C.R. a mis en place ce système afin de faciliter la gestion de la population des camps. Ce quadrillage en cellule permet de se repérer dans l'espace et de localiser le lieu d'habitation de chaque réfugié : l'attribution d'un numéro à chaque cellule puis à chaque maison tient lieu d'adresse. Mais l'objectif principal reste la circulation de l'information. Elle part du H.C.R. pour parvenir à chaque individu à travers ce système pyramidal de Comité. Un système d'adduction d'eau est mis en place dans chaque groupement. Différents lieux de culte représentant les Églises présentes dans le camp sont disséminés dans l'espace.

La nourriture est distribuée une fois par mois à chaque famille habitant dans le camp. Le H.C.R. distribue, par

${ }^{5}$ Chiffres H.C.R., communication personnelle, septembre 2003.

${ }^{6}$ Le Comité est normalement élu au suffrage universel mais dans les faits, nommé sur proposition des hommes de la cellule. l'intermédiaire du Programme Alimentaire Mondial (P.A.M.), une ration alimentaire censée couvrir l'ensemble des besoins nutritionnels. Composée de biens alimentaires (farine, haricots, sel, etc.), elle peut être complétée par d'autres produits tels que du savon, du bois de chauffe ou des semences en cas d'arrivage occasionnel. Les biens non distribués par l'aide humanitaire peuvent être achetés sur le petit marché qui se trouve à quelques mètres de l'entrée du camp. Plusieurs vendeurs proposent différents produits issus de la récolte des champs ou de la cueillette. Du poisson acheté à la chambre froide de Kimpese et des beignets confectionnés sur place complètent l'offre. La boutique tenue par l'actuel président du Comité des Réfugiés est la plus grande échoppe. Elle propose des biens plus sophistiqués : produits de beauté et de toilette, boîtes de conserve, piles, cigarettes, biscuits, bonbons, bobines de fil, etc. D'autres stands tenus par des Congolais proposent des lampes de poche, des ustensiles de cuisine, des vêtements d'occasion. Les jours de distribution de nourriture, le marché est plus animé et les stands sont plus nombreux. Certains réfugiés vendent ou troquent une partie de leur ration alimentaire contre des biens qui ne sont pas fournis par le H.C.R.. Dans le camp, de petites échoppes de tailleur, de menuisier, de coiffeur sont tenues par des réfugiés. Mais l'espace du camp est aussi façonné et marqué par la présence des agences humanitaires et de leurs sigles. Les bidons d'huile ou les sacs de farine et de haricots distribués par le P.A.M. sont recyclés en portes ou en toitures. Les bâtiments des agences humanitaires sont reconnaissables à la manière dont ils sont construits: des bâches à leurs couleurs recouvrent les toits, des drapeaux flottent à leur entrée.

Le système éducatif est pris en charge par une O.N.G. congolaise, Actions et Interventions pour le Développement et l'Encadrement Social (A.I.D.E.S.), et suit le cursus congolais. La scolarisation est assurée 
pour les enfants réfugiés et ceux des ayantdroits $^{7}$ jusqu'au cycle secondaire. Les professeurs sont soit issus du corps professoral congolais, soit sont des réfugiés qui ont tous effectué leurs études en R.D.C. En 2000, une école maternelle a été créée et accueille 250 enfants entre trois et cinq ans. Les réfugiés qui y travaillent sont employés par I.R.C. Ils reçoivent une compensation financière qui n'est pas un salaire puisque le travail des réfugiés est interdit par la loi.

Le système médical est géré par l'I.R.C. Il inclut un dispensaire qui permet d'assurer les premiers soins et d'autres services: une maternité, un programme de prévention du SIDA et des maladies sexuellement transmissibles, un autre pour le dépistage de la tuberculose, un centre nutritionnel thérapeutique pour les enfants sous-alimentés et une pharmacie distribuant les médicaments gratuitement aux malades pendant leur traitement. Les agents de santé communautaires complètent le dispositif. Ils ont pour rôle de sensibiliser la population à différentes thématiques: lutte contre les maladies sexuellement transmissibles, hygiène, vaccination... Dans ce but, ils sillonnent le camp et visitent les ménages. Ce sont des réfugiés embauchés par l'I.R.C. selon le même mécanisme que ceux employés pour assurer le fonctionnement de l'école. Parallèlement, des médecins traditionnels des villages alentours sont consultés par les réfugiés. Deux autres pharmacies tenues par des réfugiés et approvisionnées par des achats à Kimpese ou par la revente de médicaments obtenus au dispensaire, ont fonctionné avant que leurs propriétaires ne soient rapatriés.

Tous ces programmes ne satisfont pas seulement des besoins vitaux mais remplissent également plusieurs fonctions secondaires. Ils créent des espaces de socialisation, notamment par les infrastructures nécessaires à leur bon déroulement, qui renforcent le label de

${ }^{7}$ Les ayants-droits sont les propriétaires congolais des terres sur lesquelles le camp de Nkondo s'est établi.
« réfugié » pour reprendre la terminologie de Zetter (1991). Ces infrastructures constituent un lieu de passage et de regroupement des réfugiés. En effet, ces derniers savent qu'ils peuvent y trouver les membres du comité des Réfugiés car la plupart travaillent pour les agences humanitaires du camp. Ils s'y rendent lorsqu'ils souhaitent s'entretenir avec l'un d'eux. Ils y organisent aussi des réunions informelles, par exemple pour préparer leurs entrevues avec le personnel humanitaire et s'accorder sur une position commune. Ces bâtiments sont réunis à l'entrée du camp pour des raisons sécuritaires : ainsi, le personnel des agences peut être facilement évacué en cas de besoin. Les tentatives de mettre sur pied des endroits de rencontre dédiés aux réfugiés ont échoué. Le foyer social devait jouer ce rôle pour les femmes mais cela n'a pas fonctionné compte tenu de sa situation excentrée, à plusieurs centaines de mètres du centre névralgique du camp, constitué par le marché et les bâtiments des agences humanitaires. Ces derniers sont utilisés le soir venu comme lieu de rencontre par les membres du Comité des Réfugiés qui y discutent bien souvent des positions à tenir face à des décisions du H.C.R. ou des O.N.G. qui ne leur conviennent pas.

Ainsi, l'espace de travail des institutions d'aide et celui de socialisation des réfugiés sont identiques, mais ils sont utilisés à des fins différentes et quelquefois contradictoires. Ne pouvant pas construire leurs propres lieux (manque de moyens, de place, de temps, incertitude quant à la durée de leur séjour), les réfugiés s'en sont réappropriés certains pourtant clairement identifiables et identifiés comme appartenant aux agences d'aide. Mais ces lieux ne sont pas connotés et leur appartenance, revendiquée ou du moins susceptible de l'être, change selon les acteurs qui les occupent et les buts qu'ils poursuivent. Leur sens identitaire existe mais il n'est pas figé. 


\section{« Réfugié ", un label à acquérir, à utiliser et à oublier...}

Les agences humanitaires ont organisé l'espace afin de gérer la population du camp, mais elles ont aussi catégorisé les individus, notamment par l'intermédiaire de leurs programmes. Par exemple, les personnes participant aux Comités des Réfugiés forment un groupe social spécifique. Elles sont en lien avec les employés des institutions gérant le camp et ont ainsi acquis une certaine visibilité en tant que «réfugiées». Cela signifie notamment qu'elles sont identifiées en tant que telles et reconnues ainsi à l'extérieur du camp, tant par les institutions humanitaires que villageoises. D'une part, leur accès privilégié à l'information des agences d'aide et d'autre part, leur contact direct avec les employés leur permet d'acquérir un certain prestige et surtout des avantages matériels (comme la possibilité d'aller à Kimpese dans le véhicule d'une O.N.G.) ou symboliques (la fréquentation des instances dirigeantes du camp). Les autres réfugiés du camp sont moins clairement rendus visibles et jouent moins sur les bénéfices de cette étiquette.

Les populations angolaise et congolaise ont traversé la frontière au gré des guerres, des opportunités économiques ou pour raisons familiales. Il y a donc une longue tradition d'établissement des Angolais en R.D.C. et de Congolais en Angola. Certaines personnes habitant dans le camp avaient déjà vécu dans la région et ont choisi de s'établir à Nkondo pour diverses raisons, généralement en lien avec la distribution de nourriture, l'éducation gratuite et l'accès aux soins. Certains ont même fait le choix de ne pas y habiter de façon permanente. Ils n'y viennent qu'à certaines occasions, par exemple, les jours de distribution de nourriture. Ainsi, cette partie de la population joue sur deux identités, celle de réfugié et celle de villageois. Ils se revendiquent réfugiés uniquement quand cela est utile.
Une autre partie des réfugiés «surinvestit» au contraire ce label. Ce sont notamment ceux employés par les organismes d'aide. Malgré leur faible nombre, ils ont un pouvoir important. Ils sont payés en argent liquide et non pas rémunérés contre des biens ou de la nourriture, ce qui leur permet de faire office de «banquiers». Ils peuvent par exemple avancer l'argent nécessaire à l'ouverture d'un petit commerce. De plus, ils établissent des liens privilégiés avec les employés des O.N.G. dont on a vu qu'ils constituent une ressource matérielle et un capital symbolique. Certains membres du Comité des Réfugiés, après leur élection, sont embauchés par les O.N.G. afin qu'ils puissent être plus disponibles pour leurs nouvelles responsabilités. En effet, le H.C.R. estime qu'il est difficile d'occuper un tel poste et d'exercer une activité agricole en même temps. Les employer les rend disponibles à tout moment de la journée pour régler des questions relatives à la gestion du camp. Le groupe constitué par les réfugiés embauchés au sein des agences d'aide forme une des catégories les plus visibles de la population de Nkondo.

Pour attribuer les produits aux plus nécessiteux, les agences humanitaires ont opéré une classification suivant des normes économiques, sociales ou physiques, et qui ouvre droit à certains avantages en nature. Sont considérées comme «vulnérables » les femmes chefs de ménage, les personnes âgées, les handicapés physiques, etc. Elles se sont vues attribuer des cases près du foyer social, ceci devant faciliter l'intervention du personnel médico-social et rompre leur supposé isolement. Elles sont prioritaires dans l'attribution de certains biens et dans l'accès à certains programmes. Paradoxalement, il est donc finalement avantageux d'être défini comme «vulnérable ». Mais cette catégorie opérationnelle aux yeux du H.C.R. ne l'est pas pour les réfugiés. Ils considèrent que tous sont vulnérables, puisque réfugiés. De nombreuses revendications visant à son 
élimination ont eu lieu, notamment lorsque les «vulnérables» sont épargnés par les diminutions des rations alimentaires distribuées par le H.C.R.

Hormis les programmes destinés à gérer la population des camps, les projets des O.N.G. cherchent à améliorer la situation matérielle des réfugiés. Les activités génératrices de revenus ont permis la mise en place de nombreuses activités économiques par des prêts: ateliers de tailleur, restaurant, menuiserie, petits commerces, etc. De même, les projets de formation en «coupe-couture» et en menuiserie permettent l'acquisition d'un savoir-faire.

Ces catégories créées dans le but de faciliter l'administration du camp se sont intégrées de manière plus large dans son fonctionnement socio-économique, permettant ainsi l'émergence de divers groupes sociaux dont les fonctions dépassent le strict cadre des nécessités des O.N.G.

\section{Le chercheur et l'institution, une place à trouver, des méthodes à adapter}

Comment travailler en tant que chercheur dans un contexte où les relations sociales sont modelées par la présence d'une institution qui, de plus, est garante de son accès au terrain ? Comme le souligne Liisa Malkki (1995) qui a conduit une recherche sur les réfugiés Hutu en Tanzanie, à la fois en ville et dans un camp, le camp est un endroit fermé où il est relativement aisé de mener une enquête de terrain, en termes pratiques tout du moins. Néanmoins, elle reconnait que les endroits où faire de l'observation participante dépendent de nombreux facteurs qui ne sont pas liés au bon vouloir des chercheurs, mais à des circonstances logistiques : sécurité, peur du qu'en dira-t-on, etc. La première difficulté logistique à contourner est l'obtention d'une permission de recherche de la part du
H.C.R. En effet, le camp de Nkondo est un camp fermé, géré par le H.C.R, même si la gestion en est déléguée à l'O.N.G. I.R.C. Il est indispensable, pour y pénétrer en tant que chercheuse, d'obtenir une autorisation, ce qui ne va pas forcément de soi. Les agences travaillant dans les camps de réfugiés acceptent difficilement de voir une personne extérieure jauger et juger leur propre travail. Elles estiment aussi avoir déjà leurs propres analystes. En effet, l'anthropologue n'est plus l'unique récipiendaire des récits de vie des réfugiés et le seul producteur de connaissances sur le milieu humanitaire. Les journalistes, mais aussi les employés des agences d'aide, entrent en concurrence avec lui. De fait, le recueil de témoignages devient désormais indispensable pour collecter des fonds, justifier son action ou alerter l'opinion publique.

L'accès au terrain est donc largement conditionné par cette modalité et cela, tout d'abord, pour des raisons logistiques. Les déplacements jusqu'au lieu du camp se font grâce aux véhicules des agences humanitaires. J'ai utilisé dans un premier temps ceux du H.C.R. puis, par la suite, le bus de l'I.R.C. qui amenait tous les jours les personnes travaillant dans le camp. En effet, les venues des employés du H.C.R. étaient trop irrégulières pour permettre un travail de terrain efficace. Cela signifie que je n'étais pas maitre de mon temps d'enquête mais tributaire des horaires de travail des employés. Je devais également respecter certaines consignes de sécurité. Par exemple, il était impossible de rester la nuit dans le camp. Aussi, toute une partie de la vie du camp m'est-elle restée inconnue!

Le fait d'obtenir une autorisation de recherche du H.C.R. n'a pas seulement eu des conséquences sur l'organisation de mon travail. Les employés, sachant cela, s'interrogeaient sur la véritable nature de mes liens avec le siège du H.C.R. de Kinshasa qui m'avait délivré cette autorisation. La question de savoir si j'étais 
envoyée pour mener une évaluation de leur travail de manière clandestine a, dans un premier temps, tendu mes relations avec eux. Ma position pouvait, en effet, être ambigüe. Il est rare de voir des étudiants accéder aux camps. De fait, j'étais la première étudiante à entrer à Nkondo. De plus, de nombreuses études sont conduites par les équipes du H.C.R., envoyées par la délégation nationale, régionale ou même par le siège de Genève. J'ai assisté lors de mon séjour à deux visites de ce type, l'une portant sur les camps de réfugiés en Afrique centrale et l'autre, sur l'éducation. Il aurait donc été crédible que je sois une experte mandatée par un des bureaux nationaux ou régionaux. Pourtant, aucune contrepartie ne m'a été demandée en échange de l'accès au terrain. Lors de mon retour à Kinshasa, j'ai tout de même présenté brièvement quelques-uns de mes résultats, notamment concernant l'économie du camp basée sur l'endettement: les réfugiés achetaient à crédit certains biens, remboursés le mois suivant grâce aux rations fournies par le H.C.R. Toute diminution de ces rations les mettait en difficulté économique puisque les modifications ne leur étaient annoncées que quelques jours avant la distribution. J'ai ainsi tenté de satisfaire une obligation morale de rendu de résultats pour le siège tout en essayant de ne pas trahir la confiance des gens qui avaient accepté de me parler. J'ai donc choisi d'axer ma communication de manière à ce que les retombées soient positives pour les deux parties.

La présence de l'institution sur le terrain influe également sur le type de relations développées avec les protagonistes de la recherche. Dans les premiers temps, il y a une méfiance de la part des employés qu'il faut essayer de dépasser. Elle existe également du côté des réfugiés. Eux aussi se demandaient si je n'étais pas une employée d'une des organisations humanitaires. Ils ont même cru que j'étais la nouvelle responsable du bureau local du H.C.R. car je suis arrivée au moment de sa prise de fonction. C'est par mon attitude et en répétant mes explications que j'ai pu faire admettre ma position d'étudiante qui cherchait à connaitre leurs conditions de vie. En effet, je passais la journée entière dans le camp à discuter avec les réfugiés, ce qui n'est pas le quotidien des employés des agences d'aide qui font des visites occasionnelles d'une ou deux heures dans le camp. Pour autant, les réfugiés savaient que je pouvais aller et venir dans Nkondo parce que les agences humanitaires, notamment le H.C.R., l'avaient accepté et donc que j'avais un statut privilégié, tout du moins des facilités d'accès à leurs employés et à leurs infrastructures. Je me suis ainsi trouvée sous le double regard des réfugiés et des employés des organismes d'aide qui tous avaient certaines idées sur les raisons de ma présence ici et y projetaient certaines attentes, certaines peurs, certains désirs.

Au cours de mon séjour à Nkondo, j’ai eu de nombreuses conversations informelles, individuelles ou de groupe, et quelques entretiens plus en profondeur avec les réfugiés que je connaissais le mieux ou qui avaient accepté de me parler. J'ai essuyé certains refus que j'ai respectés. Je ne voulais pas que les réfugiés se sentent obligés de me parler, par exemple parce que le Président du Comité des réfugiés ou un travailleur humanitaire l'aurait demandé. Je ne pense pas que des entretiens réalisés dans ces conditions soient fructueux et il est de toute façon compréhensible que les personnes ne souhaitent pas me parler: les entretiens peuvent faire rejaillir des souvenirs douloureux liés à la fuite et la situation qu'ils vivent désormais est souvent ressentie comme tout aussi pénible. Il n'est pas facile de se retrouver en camp, dépendants de l'aide pour subvenir à ses besoins et à ceux de sa famille. Les conditions de vie sont précaires. Beaucoup habitent dans des cases de branchage et il leur manque les biens essentiels. Certains n'avaient simplement pas de temps à me consacrer: les travaux des champs leur prennent une bonne partie de la journée, surtout quand le terrain est éloigné, et le reste du temps est consacré aux tâches domestiques. Enfin, d'autres n'en voyaient 
simplement pas la nécessité ce qui est aussi parfaitement compréhensible!

Les entretiens individuels se réalisaient toujours dans le camp, au domicile des personnes. J'estimais que c'était l'endroit où les personnes se sentaient le plus en confiance notamment lorsqu'il fallait aborder des sujets douloureux. Cela avait aussi l'avantage de la discrétion. En effet, certains m'ont fait des confidences dont je pense que je n'en aurais jamais été récipiendaire si l'entretien avait eu lieu dans un endroit public. Quant aux entretiens collectifs, ils ont eu lieu, selon les circonstances, dans l'une des boutiques du marché ou dans un des complexes humanitaires du camp. J'ai également assisté à des réunions de certains Comités, notamment le Comité des Mamans, qui m'ont permis de recueillir des informations et d'organiser des entretiens collectifs. Les réunions entre les réfugiés et le personnel humanitaire, par exemple, à l'occasion d'annonces de changements dans les programmes du camp, ont aussi été riches en informations.

Afin de faciliter mon intégration dans le camp et de diminuer les tensions que je pensais inhérente à ma venue, je suis entrée par le biais d'un « interlocuteur privilégié », le président du Comité des réfugiés. Je me suis tout d'abord présentée à lui pour des raisons de convenance: de par son statut, il me semblait indispensable de lui expliquer les raisons de ma présence dans le camp. Il s'est avéré au fil du temps que celui-ci était une personne-ressource précieuse : il m'a fourni de nombreuses informations sur la vie quotidienne du camp et m'a introduit auprès de personnes intéressantes pour ma recherche, ce qui a très certainement facilité les contacts avec ces derniers. Sa boutique constituait aussi un lieu de passage et d'attente pour de nombreux réfugiés et j'ai ainsi pu organiser de manière informelle de nombreux entretiens de groupe.

\section{Une triple sensibilité du terrain à prendre en compte et à respecter}

À la lumière des conditions de l'enquête que je viens d'évoquer, effectuer une ethnographie des camps de réfugiés place le travail sous le signe d'une triple sensibilité que je vais détailler. La souffrance endurée par la population, lors de la fuite et à cause de leurs conditions de vie actuelles, en constitue une première caractéristique incontournable. Les enjeux socio-politiques inhérents à ce type de terrain forment le deuxième trait distinctif: ils sont liés aux causes du conflit qui ont conduit les réfugiés à fuir, mais aussi aux solutions politiques proposées pour résoudre la situation. Enfin, l'adaptation constante des méthodes classiques de recueil des données est la troisième particularité. Elle se fait par rapport à l'institution : le chercheur doit se positionner par rapport à elle, afin de ne pas être confondu avec ses intérêts, son agenda, son personnel, comme nous venons de le voir, mais aussi par rapport au stigmate du réfugié. Comment cette triple sensibilité modèle la collecte de données et son interprétation?

Tout d'abord, les témoignages sont recueillis désormais en vue de différentes utilisations : académiques, mais aussi journalistiques, pour illustrer des rapports d'activité ou recueillir des fonds. Pour le chercheur en sciences sociales qui tente de s'approcher le plus près possible de la "vérité » (nous en verrons plus tard les enjeux et écueils), le risque est grand d'obtenir des discours uniformisés. Les réfugiés imaginent qu'un certain type de témoignage est attendu d'eux, bien souvent misérabiliste, où ils se présentent comme passifs, un témoignage qui correspond à certaines attentes des experts, journalistes ou humanitaires.

Cette identification du chercheur en sciences sociales au système humanitaire peut créer plusieurs biais. Cela fait resurgir des souvenirs douloureux, ce qui n'est pas le 
but de l'anthropologue, que ce soit d'un point de vue éthique ou scientifique. Me concernant, je ne souhaitais évidemment pas mettre les personnes avec qui je m'entretenais dans une situation inconfortable, d'autant plus que cela n'était pas «utile», puisque mon sujet d'études portait sur la vie dans les camps et non sur l'exil. Il est tout de même impossible de se tenir à l'écart de thématiques douloureuses. Dans mon cas, le trauma n'était pas tellement celui de la fuite. Les réfugiés vont et viennent de chaque côté de la frontière au gré des conflits en cours dans l'un ou l'autre des pays. La constitution de réseaux d'entraide au fur et à mesure des années en amortit les difficultés et permet de développer des tactiques d'intégration ou tout du moins d'adaptation. Les réfugiés me parlaient plus souvent du traumatisme que constituait la vie dans le camp: le fait de vivre dans des huttes de paille, d'être dépendants de dons externes sans pouvoir assurer eux-mêmes la subsistance de leur famille ou d'être labellisés comme réfugiés étaient les motifs les plus courants de doléances. Ces situations étaient d'autant plus mal vécues que ces personnes avaient connu d'autres conditions de vie en R.D.C. et avaient l'impression d'avoir été «trompées » par les avantages présumés de la vie en camps : gratuité des soins et de la scolarité, distribution de nourriture entre autres. Cela induit aussi des requêtes et des attentes que le chercheur n'est pas forcément en position de remplir ${ }^{8}$. Pourtant, comme le souligne Hammond (2004), le chercheur a une nécessité morale d'utiliser son accès privilégié au H.C.R. ou aux O.N.G. pour améliorer les conditions de vie ou relayer les revendications des réfugiés. L'équilibre à trouver pour obtenir autre chose qu'un discours préfabriqué est donc fragile.

Je souhaite revenir maintenant sur les enjeux autour de la "vérité », tant pour le

${ }^{8} \mathrm{Je}$ renvoie ici à l'article de Julie Baujard (2005) pour une revue détaillée des attentes induites par la position du chercheur. chercheur que pour le réfugié. En ce qui concerne ce dernier, il est compliqué de se confier. Pour Knudsen (1995), la confiance des réfugiés est érodée par leur fuite: certains ont dû faire face à la trahison de proches et se retrouvent ensuite dans un camp où ils doivent s'en remettre à des étrangers pour assurer leur survie quotidienne. Il existe donc des tensions entre les différentes options qui s'offrent: dire la "vérité », c'est-à-dire se livrer et livrer son intimité, le plus souvent à un étranger, ou bien « arranger la vérité » en délivrant un discours que l'on pense attendu par l'interlocuteur, en se composant une histoire, une identité plus conforme à ses attentes ou encore en optant pour une présentation stratégique de soi, correspondant à l'image que l'on souhaite donner et qui peut permettre d'atteindre certains objectifs. Les récits sont finalement le résultat d'un dosage entre ces diverses options. Le chercheur, lui, souhaite s'approcher au plus près de la "vérité ». Pourtant, cela ne fait pas sens. Comme vu plus haut, le réfugié livre une version des faits qui est un mélange entre plusieurs options. Comme le souligne Malkki (1995), recueillir des discours stéréotypés est aussi riche d'enseignements, notamment sur les relations entre les réfugiés et les humanitaires, et tout savoir ne signifie pas que l'on est au plus proche du vécu des gens. Chacun a droit à garder une part de secret, cela ne rend pas l'enquête moins valable, et ce que les gens cachent n'est pas forcément le plus intéressant: anthropologue ne signifie pas voyeur.

De plus, comme Powles (2004) le souligne, la construction du récit autour des faits de vie renseigne et permet de tirer des analyses bien plus riches que la connaissance de faits bruts. Elle fait partie de la construction de l'histoire du réfugié et de la façon dont il se présente et se voit. Elle explique qu'il faut ainsi faire confiance à l'informateur qui tente de rendre compte de sa propre vie de la manière qu'il pense la plus fidèle. Elle estime que la confiance et les bonnes relations sont essentielles dans la 
constitution d'une relation d'enquête fructueuse. Par cela, elle entend que l'informant doit savoir qui est le chercheur: quelle est sa place et quelles sont ses intentions, en échange celui-ci doit montrer son "intérêt», sa «bonne volonté, sa "sensibilité ». Nous pouvons aller plus loin quand nous considérons le rôle de la confiance dans la relation entre enquêté et anthropologue. Elle est en effet primordiale, voire centrale. Elle est souvent envisagée comme celle que place l'enquêté dans le chercheur en sciences sociales, mais il me semble que le chercheur doit également être capable de s'en remettre à l'enquêté et à sa connaissance privilégiée du terrain. Mon informateur privilégié avait bien compris le but de ma présence à Nkondo et m'a guidé à travers les méandres du camp, m’indiquant des personnes intéressantes à rencontrer ou des réunions où je devrais aller. En m'en remettant à lui, en acceptant de modifier mon programme pour suivre ses suggestions ou en prenant en compte ses remarques, la confiance s'est renforcée graduellement entre nous. Ce processus de "lâcher-prise » m'a permis l'accès à de nombreuses informations qu'ignoraient pour la plupart les employés des O.N.G.

Enfin, une dernière difficulté concerne la temporalité et la notion d'urgence. Le camp de Nkondo fut fermé le 31 décembre 2004. Il est donc impossible désormais d'y retourner et de recouper les récits ou d'obtenir des informations manquantes. Les réfugiés sont retournés chez eux, pour certains, se sont installés dans d'autres provinces angolaises, pour d'autres. Certains sont également restés en R.D.C. Le stigmate de réfugié n'est pas toujours facile à porter une fois rentrés en Angola. Des tensions existent entre les personnes qui sont restées en Angola durant la guerre et celles qui ont fui le pays. Ainsi, certains ne souhaitent plus être identifiés en tant que tels.
Conclusion

Le camp est un espace dessiné par les agences d'aide que les réfugiés s'approprient. Celles-ci mettent en place différentes catégories pour faciliter la gestion de la population. Le label de "réfugié » et ceux distribués au travers des programmes d'aide, peuvent constituer pour qui sait s'en servir un capital social voire un capital tout court, non seulement dans le camp mais également à l'extérieur. Le travail du chercheur s'inscrit dans ce contexte particulier. Nous l'avons vu, la présence de l'institution est centrale et modèle les relations sociales, entre employés des agences d'aide et réfugiés, mais aussi entre réfugiés et entre réfugiés et chercheur. Elle constitue une donnée avec laquelle ce dernier doit composer. Le chercheur doit également faire face à certaines difficultés dues aux parcours douloureux des exilés : ne pas raviver des traumatismes tout en parvenant à recueillir des histoires de vie requiert une certaine éthique. La confiance entre enquêté et chercheur est alors primordiale. Elle doit fonctionner pour cela dans les deux sens. Face à ces différents obstacles, le chercheur doit mettre en place de nouvelles modalités de recherche. Pour autant, ces obstacles rendent le recueil d'informations plus intense et permettent au chercheur de développer de nouvelles méthodes d'investigation et ainsi d'enrichir son répertoire méthodologique.

Virginie Tallio

Anthropologue

Centro de Estudos Africanos-IUL ISCTE-Instituto Universitário de Lisboa virginie.tallio@gmail.com 


\section{Bibliographie}

Baujard, Julie (2005) Ni espionne ni avocate. La place ambigüe de l'ethnologue auprès des réfugiés in F. Bouillon, M. Fresia, V. Tallio (eds.), Terrains sensibles. Expériences actuelles de l'anthropologie, Paris, Éditions de l'EHESS, pp. 125-143.

Bouillon, Florence ; Fresia, Marion ; Tallio, Virginie (eds.) (2005) Terrains sensibles. Expériences actuelles de l'anthropologie, Paris, Éditions de l'EHESS, 208 p.

Hammond, Laura C. (2004) This place will become bome. Refugee repatriation to Ethiopia, Ithaca, Londres, Cornell University Press, 257 p.

Knudsen, John Chr. (1995) When trust is on trial. Negotiating refugees narratives, in V. E. and John Chr. Knudsen (eds.) Mistrusting refugees, Berkeley, Los Angeles, London, University of California Press, pp. 13-35.

Malkki, Liisa H. (1995) Purity and exile: violence, memory and national cosmology among Hutu refugees in Tanzania, Chicago, Chicago University Press, 374 p.
Powles, Julia (2004) Life history and personal narrative: theoretical and methodological issues relevant to research and evaluation in refugee contexts, New issues in refugee research, Working paper $\mathrm{n}^{\circ} 106$, UNHCR, Evaluation and Policy Unit.

Richardson, Anna (2002) Angola: civil war and bumanitarian crisis. Developments from mid 1999 to end 2001, UNHCR, Emergency and Security Service, $30 \mathrm{p}$.

Sabakinu, Jacob (2004) Les populations réfugiées dans l'Ouest de la République démocratique du Congo in Guichaoua, André (ed.), Exilés, réfugiés, déplacés en Afrique centrale et australe, Paris, Karthala, pp. 567629.

Zetter, Roger (1991) Labelling refugees: forming and transforming a bureaucratic identity, Journal of Refugee Studies, vol. 4, $\mathrm{n}^{\circ} 1$, pp. 39-62. 